\title{
A feasibility study of postoperative chemotherapy with S-1 and cisplatin (CDDP) for gastric carcinoma (CCOG0703)
}

\author{
Yasuhiro Kodera ${ }^{1}$, Akiharu IshiYama ${ }^{2}$, Takaki Yoshikawa ${ }^{3}$, Takashi Kinoshita ${ }^{4}$, Seiji Ito ${ }^{5}$, \\ Hiroyuki Yokoyama ${ }^{6}$, Yoshinari Mochizuki ${ }^{6}$, Hiroaki Ito ${ }^{7}$, Akira Tsuburaya ${ }^{3}$, Junichi Sakamoto ${ }^{8}$, \\ and Akimasa NAKAO ${ }^{1}$ for the Chubu Clinical Cancer Group \\ ${ }^{1}$ Department of Surgery II, Nagoya University Graduate School of Medicine, 65 Tsurumai-cho, Showa-ku, Nagoya 466-8550, Japan \\ ${ }^{2}$ Department of Surgery, Okazaki City Hospital, Okazaki, Japan \\ ${ }^{3}$ Department of Surgery, Kanagawa Cancer Center, Yokohama, Japan \\ ${ }^{4}$ Department of Surgery, Yokkaichi Municipal Hospital, Yokkaichi, Japan \\ ${ }^{5}$ Department of Gastroenterological Surgery, Aichi Cancer Center Hospital, Nagoya, Japan \\ ${ }^{6}$ Department of Surgery, Komaki Municipal Hospital, Komaki, Japan \\ ${ }^{7}$ Department of Surgery, Tajimi Hospital, Tajimi, Japan \\ ${ }^{8}$ Young Leaders Program, Nagoya University Graduate School of Medicine, Nagoya, Japan
}

\begin{abstract}
Background. The outcome of stage III gastric cancer patients treated by $\mathrm{D} 2$ dissection followed by adjuvant chemotherapy with S-1 remains unsatisfactory. Moreover, some patients with a preoperative diagnosis of stage II/III turn out to be stage IV after surgical exploration, and a standard postoperative treatment for this population has not been established.

Methods. A feasibility study of postoperative S-1/cisplatin (CDDP) was performed with patients who underwent gastrectomy for what turned out to be a stage IV gastric cancer. The primary endpoint of the trial was the relative dose intensity during five courses of S-1/CDDP. Several criteria to skip, postpone, or reduce the dose had been predetermined.

Results. Between 2007 and 2009, 31 patients were accrued, including 19 patients who were positive for peritoneal washing cytology, 6 with peritoneal seeding, 5 with metastasis to the paraaortic nodes, and 4 with other distant metastases. Only 7 patients completed five cycles as planned (median, two cycles). The median relative dose intensities of S-1 and CDDP were $37 \%$ and $40 \%$, respectively. Causes of treatment failure were failure to fulfill criteria for starting a new course within 5 weeks of the last administration of $\mathrm{S}-1$ in 7 , patient refusal in 6 , disease recurrence/progression in 4 , need to reduce dose by two levels in 4, and two successive skips of CDDP in 3 patients. The median progression-free survival time of all patients was 363 days.

Conclusions. Although promising in the neoadjuvant and advanced/metastatic setting, $S-1 / C D D P$ is too toxic as a postgastrectomy treatment for Japanese patients.
\end{abstract}

Key words Gastric cancer $\cdot$ Metastasis $\cdot$ S- 1 - CDDP $\cdot$ Relative dose intensity

Offprint requests to: $\mathrm{Y}$. Kodera

Received: March 10, 2010 / Accepted: May 19, 2010

\section{Introduction}

Gastric carcinoma is the second most common cause of cancer-related death worldwide [1], and remains a major health problem in the Far East. The survival of patients with gastric cancer is often dismal even if treated with potentially curative resection, and various perioperative therapies directed against micrometastases have been proposed and delivered in addition to improve the outcome [2-4].

In Japan, S-1 (1 M tegafur- 0.4 M gimestat- 1 M otastat potassium) has become a key drug in the treatment of gastric cancer. It was found to be remarkably active as a single agent in the treatment of unresectable/metastatic gastric cancer [5,6], while the response rate was shown to be further enhanced by the addition of cisplatin (CDDP), exceeding 70\% in a phase II trial [7]. A phase III trial has shown that single-agent S-1 administered postoperatively for 1 year significantly improves the outcome of patients with stage II/III gastric cancer over treatment with surgery alone [4]. Another phase III trial has shown the benefit of S-1/CDDP over S-1 in the treatment of unresectable/metastatic cancer [8]. These facts point to a strategy of administering S-1/ CDDP instead of S-1 alone as an adjuvant therapy for the further improvement of outcome in patients with resectable disease, although the gastrointestinal toxicity of this combination casts doubt as to its feasibility when delivered postoperatively.

Advanced gastric cancer is often associated with peritoneal metastasis. Current imaging studies rarely detect peritoneal deposits, although the detection of even minute amounts of cancer, such as those represented by positive cytology of peritoneal washes, usually renders the cancer incurable [9]. This knowledge 
prompted several investigators to perform staging laparoscopy prior to radical surgery [10]. However, it is not currently considered as a part of routine preoperative workup in Japan, where gastric cancer is one of the commonest types of cancer and is often treated at community hospitals in the hands of general surgeons. Consequently, a certain proportion of patients for whom curative surgery had been planned turn out to have a stage IV disease at surgery, in the form of peritoneal deposits and positive washing cytology. When peritoneal deposits are found to be minimal, these patients usually undergo resection as planned, but no evidence-based strategy as a postoperative therapy exists for this population.

In the present study, the feasibility of an S-1/CDDP combination given postoperatively was evaluated, primarily to establish a standard of care for patients who undergo gastrectomy for stage IV disease. In addition, we had an intention to test this combination as a candidate for novel postoperative adjuvant chemotherapy to be delivered to stage III patients, whose prognosis remain poor when treated with postoperative S-1 monotherapy, the current standard of care.

\section{Patients and methods}

\section{Patient eligibility}

Eligible patients had to meet all of the following criteria: (i) a confirmed diagnosis of gastric adenocarcinoma, (ii) age less than 75 years, (iii) gastrectomy performed within 6 weeks of initiation of chemotherapy (iv), stage IV disease according to the Japanese classification of gastric carcinoma [11] as confirmed by preoperative imaging studies and/or histopathological examinations, (v) no prior treatment besides surgery, (vi) European Cooperative Oncology Group performance status of 0 to 1 , (vii) adequate organ functions, defined as white blood cell count $4000-12000 / \mathrm{mm}^{3}$, total neutrophil count $2000 / \mathrm{mm}^{3}$ or more, platelet count $100000 / \mathrm{mm}^{3}$ or more, hemoglobin $9.0 \mathrm{~g} / \mathrm{dL}$ or more, serum creatinine within the normal range according to the criteria of the hospital where the study was performed, total serum bilirubin less than $1.5 \mathrm{mg} / \mathrm{dl}$, serum aspartate aminotransferase and alanine aminotransferase less than $100 \mathrm{IU} / \mathrm{l}$, and creatinine clearance $60 \mathrm{ml} / \mathrm{min}$ or more. Patients had to have a life expectancy of more than 3 months, with no other active malignancies or uncontrolled concomitant diseases. Written informed consent was obtained from all participants after they had received a full explanation of the nature of the study. The study was approved by the institutional review board of Nagoya University Hospital and all other hospitals belonging to the Chubu Clinical Oncology Group $(\mathrm{CCOG})$ that participated in this multicenter trial.

\section{Pretreatment evaluation, treatment plan, and dose attenuation}

At baseline, a complete medical history was taken, and a physical examination was performed. Laboratory assessment at baseline included blood cell counts, serum chemistry profiles, serum tumor markers (carcinoembryonic antigen; CA19-9), and urinalysis. Patients also underwent a baseline electrocardiographic examination and computed tomography (CT) scans of the chest, abdomen, and pelvis. At surgery, the intraperitoneal cavity was searched for peritoneal deposits and a cytologic examination of the peritoneal washes collected at the Douglas pouch was routinely performed. Histopathological detection of cancer cells by this examination is designated in the Japanese classification of gastric carcinoma [11] as CY1 and the patient is subsequently classified as stage IV.

Chemotherapy was to be started at 2-6 weeks after surgery. Patients received S-1 orally at the following doses twice daily for 3 weeks, followed by 2 weeks without chemotherapy. Patients with a body surface area of less than $1.25 \mathrm{~m}^{2}$ received $80 \mathrm{mg}$ daily; those with a body surface area of $1.25 \mathrm{~m}^{2}$ to less than $1.5 \mathrm{~m}^{2}$ received $100 \mathrm{mg}$ daily; and those with a body surface area of $1.5 \mathrm{~m}^{2}$ or greater received $120 \mathrm{mg}$ daily. This 5 -week cycle was repeated mainly in an outpatient setting. The exception was the delivery of CDDP, for which the patients were to be admitted for three nights and given continuous intravenous fluid administration with the antiemetics granisetron and dexamethasone. Five cycles of S-1/CDDP were to be delivered as a protocol treatment, after which the patients were recommended to receive further chemotherapy with single-agent S-1 in the absence of disease progression. If the patients had either hematological toxicity of grade 3 or greater, nonhematological toxicity of grade 2 or greater, serum creatinine exceeding the normal range according to the criteria of the hospital, or creatinine clearance of less than $50 \mathrm{ml} / \mathrm{min}$ during the previous course, the daily dose of S-1 was decreased from $120 \mathrm{mg}$ to $100 \mathrm{mg}$, from $100 \mathrm{mg}$ to $80 \mathrm{mg}$, or from $80 \mathrm{mg}$ to $50 \mathrm{mg}$, and the dose of CDDP was decreased by $10 \mathrm{mg} /$ $\mathrm{m}^{2}$. If the patients failed to fulfill the above criteria on day 1 of the new course, the new course was to be postponed until recovery. If such toxicity occurred on day 8, CDDP was to be skipped. Under these strict rules, the protocol treatment was to be discontinued in the event of (i) postponement of the new course for 3 weeks in a row, (ii) dose reduction of S-1 or CDDP by two levels, (iii) skipping CDDP for two cycles in a row, (iv) other adverse events that were considered unmanageable, (v) withdrawal of consent from the patient, or (vi) disease recurrence or progression. Patients who failed the treatment were allowed to be given a second- 
line chemotherapy at the discretion of the surgeons/ oncologists.

Disease status was assessed once every 3 months on the basis of serum tumor markers and at least once every 6 months by CT scanning until the completion of five cycles of treatment. Adverse events were monitored by interviews, blood chemistry profiles, and blood cell counts once every 2 weeks. All toxic effects were graded according to National Cancer Institute Common Toxicity Criteria (NCI-CTC; version 2.0).

\section{Endpoints and study design}

The primary endpoint of the study was the relative dose intensity (percentage of the dose actually administered out of the planned dose calculated from the body surface area) during five cycles of treatment with S-1/CDDP. The secondary endpoints were the proportion of patients who tolerated five cycles, safety, progression-free survival time, and overall survival time.

No data on relative dose intensity actually exist for the present combination therapy in the postoperative setting. A relative dose intensity of $80 \%$ is generally considered acceptable. Postoperative S-1/CDDP could be considered feasible for further exploration if the parameter in this study fell between $66 \%$ and $95 \%$ (95\% confidence interval when a relative dose intensity of $80 \%$ was obtained with 27 patients). Thus, the accrual of 30 patients was planned to prove this point.

\section{Results}

\section{Patient population}

Thirty-one patients were enrolled between October 2007 and February 2009. The demographic and clinicopathological characteristics of the patients are shown in Table 1 . The mean age of the patients was 61.5 years (range, 34-73 years). The male/female ratio was 22:9. Ten patients underwent distal gastrectomy and the other 21 received a total gastrectomy. Splenectomy was performed in 10 patients, of whom 1 also underwent resection of the pancreatic tail. Three patients underwent coresection of the transverse colon and 1 patient received a hepatectomy. Surgery in 19 patients was R2 resection, mostly due to peritoneal deposits, whereas surgery turned out to be R1 in 9 patients who were positive for cytologic examination of the peritoneal washes (CY1) without any other distant metastasis. Three other patients underwent $\mathrm{R} 0$ resection. One of these had been classified as stage IV due to pT4bpN2 disease but had a curative resection. One patient had metastasis to the paraaortic lymph node and underwent D3 dissection (eradication of the paraaortic lymph nodes). Another patient had a liver metastasis which was coresected.

\section{Compliance, relative dose intensity, and toxicity}

The median relative dose intensity of CDDP was $40 \%$ and that of S-1 was $37 \%$ (Fig. 1). Patients tolerated a median of two cycles of treatment, and only seven patients $(22 \%)$ completed all five cycles. The reasons for discontinuation of the treatment were failure to start S-1 within 3 weeks after the end of the previous course in seven patients, patient refusal due to adverse events in six patients, disease progression in four patients, dose reduction of S-1 or CDDP by two levels in four patients, and skipping CDDP for two cycles in a row in three patients. Time to treatment failure was 70 days (Fig. 2). Most patients (15/20; 75\%) who discontinued the designated treatment due to adverse events received chemotherapy with single-agent S-1. Only S-1 delivered as the protocol treatment was included in the calculation of relative dose intensity.

The most frequent grade 3/4 toxicity was neutropenia, observed in $29 \%$ of the patients (Table 2). Grade $3 / 4$

Table 1. Patient demographics $(n=31)$

\begin{tabular}{llc}
\hline Variables & & \\
\hline Age (years) & Mean (range) & $61.5(34-73)$ \\
Sex & Male/female & $22 / 9$ \\
PS (ECOG) & $0 / 1$ & $21 / 10$ \\
pT & T3/T41/T4b & $2 / 18 / 10$ \\
pN (JCGC) & N0/N1/N2/N3/NX & $1 / 10 / 14 / 5 / 1$ \\
pN (TNM) & N0/N1/N2/N3/NX & $1 / 2 / 7 / 20 / 1$ \\
Liver metastasis & Negative/positive & $29 / 2$ \\
Peritoneal metastasis & Negative/positive & $23 / 8$ \\
M (TNM) & M0/M1 & $15 / 16$ \\
Peritoneal washing cytology & Negative/positive & $8 / 20$ \\
\hline
\end{tabular}

PS, performance status; ECOG, Eastern Cooperative Oncology Group; JCGC, Japanese classification of gastric carcinoma; TNM, tumor node metastasis classification by the International Union Against Cancer 


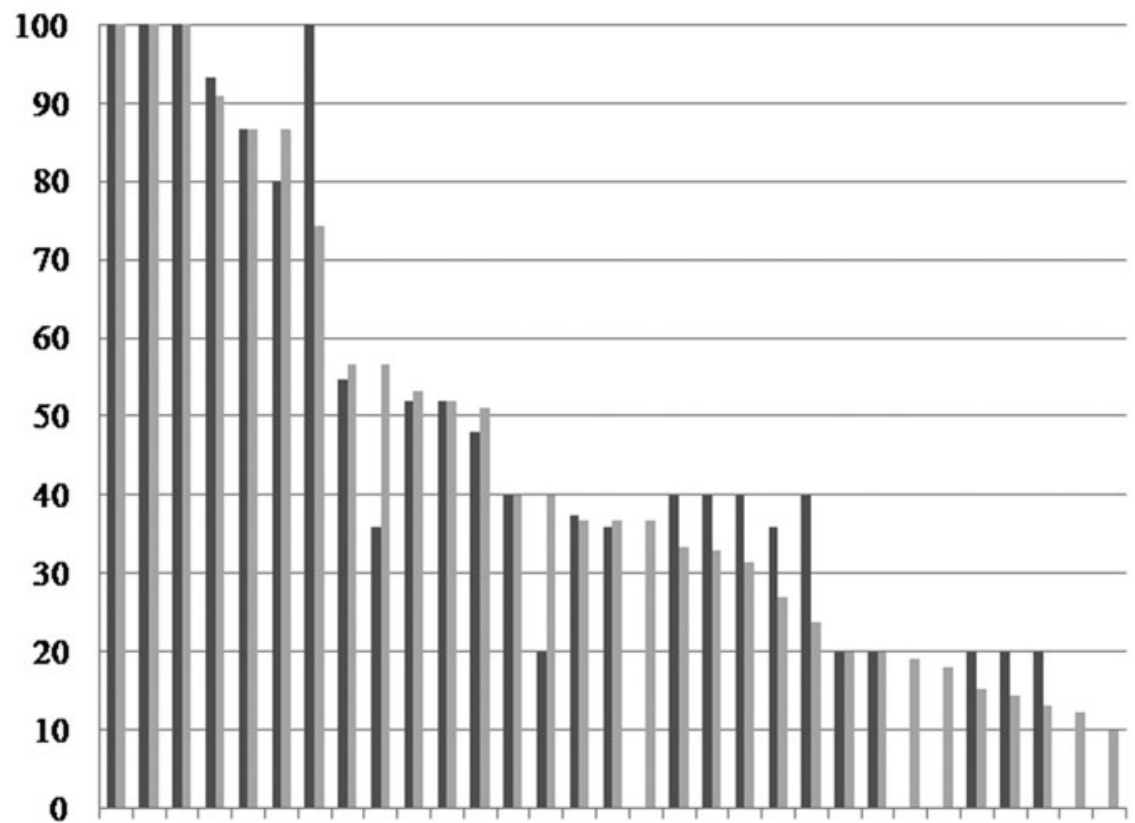

Fig. 1. Relative dose intensity of S-1 (gray bars) and cisplatin (CDDP; black bars) in each patient registered. The median relative dose intensities of S-1 and CDDP were $37 \%$ and $40 \%$, respectively. S-1 given as monotherapy after failure of the protocol treatment was excluded from the calculation of relative drug intensity

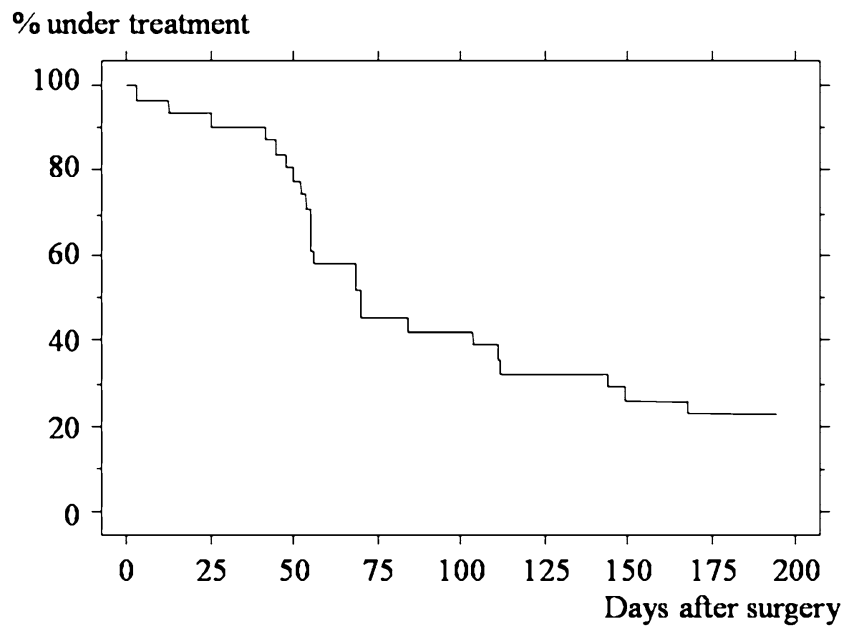

Fig. 2. Curve showing time to treatment failure. The median number of cycles delivered was two, with only $7 / 31$ patients completing the five cycles as originally planned

nonhematological toxicity included anorexia (23\%), nausea $(10 \%)$, and fatigue $(10 \%)$. Anemia of all grades was observed in $97 \%$ of the patients, possibly reflecting the fact that the treatment was given postoperatively. Anorexia and nausea of all grades was observed in $77 \%$ and $74 \%$, respectively. The most prominent skin manifestation was pigmentation, a symptom peculiar to S-1, showing diffuse darkening of skin color, which is sometimes observed in various parts of the body.

\section{Survival}

At a median follow-up time of 536 days or until death, the median progression-free survival time was
Table 2. Adverse events

\begin{tabular}{lcc}
\hline Events & All grades $(\%)$ & Grade 3 and $4(\%)$ \\
\hline Leukopenia & $21(68)$ & $2(6.5)$ \\
Neutropenia & $21(68)$ & $9(29)$ \\
Anemia & $30(97)$ & $5(16)$ \\
Thrombocytopenia & $13(42)$ & $0(0)$ \\
Anorexia & $24(77)$ & $7(23)$ \\
Nausea & $23(74)$ & $3(10)$ \\
Vomiting & $7(23)$ & $0(0)$ \\
Fatigue & $17(55)$ & $3(10)$ \\
Diarrhea & $13(42)$ & $1(3.2)$ \\
Pigmentation & $12(39)$ & $0(0)$ \\
Stomatitis & $8(26)$ & $0(0)$ \\
GOT & $13(42)$ & $0(0)$ \\
GPT & $12(39)$ & $0(0)$ \\
Bilirubin & $3(9.6)$ & $0(0)$ \\
Creatinine & $6(19)$ & $0(0)$ \\
\hline
\end{tabular}

363 days, and the median overall survival time was 813 days (Fig. 3).

\section{Discussion}

Preoperative or perioperative adjuvant therapy with cytotoxic agents with or without radiation is considered promising for the treatment of gastric cancer [2-4]. In a trial testing perioperative chemotherapy, chemotherapy was more easily delivered preoperatively than postoperatively [3], partially because patients are more vulnerable to the drug-related adverse reactions shortly after gastrectomy. Accurate staging by arrays of examinations including staging laparoscopy followed by several months of preoperative therapy is not suitable for 


\section{\% survival}

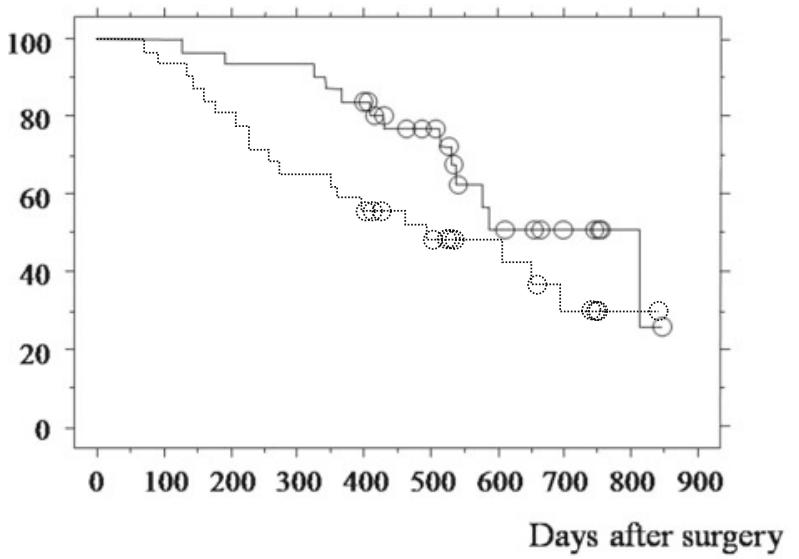

Fig. 3. Overall survival (solid line) and progression-free survival (dotted line) of all patients obtained after the mean follow up of 536 days or until death

delivery in general practice worldwide, and the standard of care in the United States, for example, is surgery followed by postoperative chemoradiation [2]. The optimal moment to perform curative surgery during the course of multimodal treatment is actually unknown, and is often decided upon according to common medical practice in each country.

Gastric cancer is more often diagnosed at an early stage in Japan compared with the timing in Western countries, due in part to nationwide screening, and this has led to a favorable outcome [12]. Early diagnosis and early treatment has therefore been considered the gold standard, and surgery is generally performed as soon as possible for fear of disease progression. Owing to the limitations in the sensitivity of preoperative imaging studies, with staging laparoscopy still not a standard practice in community hospitals, peritoneal metastases are sometimes found at surgery, either as macroscopically recognizable deposits or as micrometastases (CY1). In such circumstances, surgeons often proceed with gastrectomy as originally planned, and what is considered as the best available chemotherapy is given afterwards. Whether this strategy is superior to stomach-conserving therapy (staging laparoscopy followed by chemotherapy) is unknown, and the question is currently under investigation by a Japan Clinical Oncology Group (JCOG) randomized trial [13]. In the meantime, identification of the best available chemotherapy in the postoperative setting is warranted. It was logical to consider S-1/CDDP as a candidate for the standard of care in this setting, because this combination achieved a significantly longer median survival time in a phase III trial for advanced/metastatic gastric cancer when compared with S-1 monotherapy [8].
In the present study, the feasibility of postoperative S-1/CDDP was explored with patients who underwent surgery for stage IV disease. Another intention was that a positive result in this study may lead to the future application of this promising regimen as postoperative adjuvant therapy for stage III cancer, whose outcome remains unsatisfactory with the strategy of surgery followed by single-agent S-1. Difficulties had been anticipated, however, because the Japanese patients seemed vulnerable to various adverse events in the postoperative setting. S-1/CDDP has been feared because of its gastrointestinal and renal toxicity. Nausea and anorexia are commonly observed adverse reactions after the administration of CDDP, and dehydration due to impaired oral food intake could enhance the renal toxicity of CDDP. Despite the use of serotonin antagonists that have decreased the incidence of severe vomiting, patients already with some degree of gastrectomyrelated discomfort might suffer from even mild gastrointestinal toxicities; much more so than those who have received other types of surgery. Renal toxicity in turn affects the clearance of gimestat, a component of S-1 that inhibits the metabolism of tegafur, leading to myelotoxicity and other adverse events [14]. Actually, the toxicity profile of S-1 monotherapy observed in a phase II study for advanced/metastatic cancer [6] was far less intense compared with that reported in a feasibility study in the postoperative adjuvant setting [15]. Myelotoxicity of all grades was increased by twofold and gastrointestinal toxicity by three-old in the feasibility study. Surprisingly, patients who received the same treatment in a subsequent randomized trial comparing postoperative S-1with surgery alone in curatively resected stage II/III cancer did better [4], owing to the establishment of more precise rules regarding dose attenuation, alteration of the treatment schedules, and discontinuation of the protocol treatment.

In addition to adhering to these rules for the safe postoperative delivery of S-1, the present study paid particular attention to renal function: serum creatinine level had to be within the normal range and creatinine clearance had to be no less than $50 \mathrm{ml} / \mathrm{min}$ for a patient to continue with the treatment. Consequently, 14 patients failed to fulfill either of several criteria to continue treatment, and had to refrain from receiving all five cycles, in addition to the 6 patients who refused further treatment. No long-lasting or life-threatening toxicity occurred, and the treatment could be considered safe as long as one adhered to the protocol. On the other hand, more patients might have completed the treatment as planned had the criteria been less stringent.

Anemia was the commonest adverse event, observed in $97 \%$ of our patients, and this may have enhanced general fatigue. Anorexia, nausea, and fatigue were the 
commonest nonhematological adverse events. Toxicities generally tended to linger on, preventing the treatment from being delivered on schedule. Surprisingly, a comparison with the phase III trial comparing S-1/CDDP with S-1 monotherapy for advanced/metastatic cancer [8] revealed that the incidences of various toxicities observed in the S-1/CDDP group of that study, including those of grade 3 or greater, were actually similar to those observed in the present study. Presumably, it took longer to recover from these toxicities to fulfill the criteria for the initiation of a new cycle of treatment among the postoperative patients. In addition, even grade 1 or 2 gastrointestinal toxicities may have significantly affected patients who had just received gastrectomy.

In the present study, discontinuation of S-1/CDDP did not mean termination of chemotherapy, because 21 of the 24 patients who could not tolerate S-1/CDDP were treated with a second-line chemotherapy. Fifteen of the 20 patients who discontinued S-1/CDDP for reasons other than disease progression were given S-1 monotherapy. Thus, most patients who were entered in the trial eventually received S-1 until progression, and this presumably led to the median progression-free survival of 363 days. In another trial, the authors evaluated the efficacy of S-1 monotherapy among patients with free cancer cells in the peritoneal cavity (CY1 stage patients) treated with $\mathrm{R} 1$ resection [16]. Overall survival time in that trial was 705 days and progression-free survival was 496 days. With several patients treated by R2 resection included, the progression-free survival obtained in the present study could be considered as acceptable. With the relative dose intensity of less than $40 \%$, however, S-1/CDDP did not meet the expectation as a candidate for further evaluation in the postoperative setting.

S-1 being a key drug for chemotherapy for gastric cancer in Japan, one may need to turn to other combinations if S-1 alone is deemed insufficient [17-19]. Taxanes with a lower incidence of gastrointestinal toxicities may be particularly good candidates as a partner of S- 1 in the postoperative setting, although the superiority of these combinations over S-1 alone is yet to be proven by randomized trials. Another option could be oxaliplatin, which has been shown not to be noninferior to CDDP [20] and is associated with a lower incidence of gastrointestinal and renal adverse events. A combination of S-1 and oxaliplatin has been established and evaluated in a phase II trial [21], and further steps for its approval for gastric cancer treatment in Japan are awaited.

On the other hand, further attempts to reevaluate postoperative S-1/CDDP may not be futile. Aprepitant, a novel anti-emetic recommended by the National Comprehensive Cancer Network guideline that became available after the termination of the present study, could significantly improve tolerability to CDDP. In the meantime, members of the Japan Clinical Oncology Group proposed a modified version of the postoperative treatment in which the first course consisted of S-1 alone while CDDP was to be added only from the second course onwards, thus delaying the administration of CDDP. This strategy led to a significant improvement in compliance, and final analysis and publication of their data is eagerly awaited.

Of note, the combination of 5-fluorouracil (5FU) and CDDP has repeatedly failed to show prognostic benefit as a postoperative adjuvant therapy in Western countries [22, 23]. This could eventually be the fate of the S-1/CDDP combination given postoperatively. S-1/ CDDP can be administered more easily in the neoadjuvant setting $[24,25]$, and this strategy is currently being explored in the JCOG0501 study (a phase III trial comparing a group treated by two to three courses of S-1/ CDDP followed by surgery and S-1 monotherapy with a group treated by surgery followed by S-1 monotherapy) in a population with linitis plastica-type cancer. This trial could result in a paradigm shift in Japan, after which more patients with newly diagnosed advanced cancer would initially undergo chemotherapy.

To conclude, the combination of S- 1 and CDDP in the dose and schedule used in the present study cannot be recommended as a candidate for postoperative chemotherapy in Japanese patients with gastric cancer. The use of less toxic drug combinations and a greater focus on neoadjuvant chemotherapy are among future strategies to improve the outcome of advanced gastric cancer.

\section{References}

1. Pisani P, Parkin D, Bray F, Ferlay J. Estimates of the worldwide mortality from 25 cancers in 1990. Int J Cancer 1999;83:18-29.

2. Macdonald J, Smalley S, Benedetti J, Hundahl SA, Estes NC, Stemmermann GN, et al. Chemoradiotherapy after surgery compared with surgery alone for adenocarcinoma of the stomach or gastroesophageal junction. N Engl J Med 2001;345:725-30.

3. Cunningham D, Allum W, Stenning S, Thompson JN, Van de Velde $\mathrm{CJ}$, Nicolson M, et al. Perioperative chemotherapy versus surgery alone for resectable gastroesophageal cancer. N Engl J Med 2006;355:11-20.

4. Sakuramoto S, Sasako M, Yamaguchi T, Kinoshita T, Fujii M, Nashimoto A, et al. Adjuvant chemotherapy for gastric cancer with S-1, an oral fluoropyrimidine. N Engl J Med 2007;357: 1810-20.

5. Koizumi W, Kurihara M, Nakano S, Hasegawa K. Phase II study of S-1, a novel oral derivative of 5-fluorouracil, in advanced gastric cancer. For the S-1 Cooperative Gastric Cancer Study Group. Oncology 2000;58:191-7.

6. Sakata Y, Ohtsu A, Horikoshi N, Sugimachi K, Mitachi Y, Taguchi T. Late phase II study of novel oral fluoropyrimidine anticancer drug S-1 (1 M tegafur-0.4 M gimestat-1 $\mathrm{M}$ otastat potassium) in advanced gastric cancer patients. Eur J Cancer 1998;34:1715-20.

7. Koizumi W, Tanabe S, Saigenji K, Ohtsu A, Boku N, Nagashima F, et al. Phase I/II study of S-1 combined with cisplatin in patients with advanced gastric cancer. Br J Cancer 2003;89:2207-12. 
8. Koizumi W, Narahara H, Hara T, Takagane A, Akiya T, Takagi M, et al. S-1 plus cisplatin versus S-1 alone for first-line treatment of advanced gastric cancer (SPIRITS trial): a phase III trial. Lancet Oncol 2008;9:215-21.

9. Kodera Y. Disseminated cancer cells in the peritoneal cavity: what can we do when we detect them? Gastric Cancer 2008;11: 192-3.

10. Nakagawa S, Nashimoto A, Yabusaki H. Role of staging laparoscopy with peritoneal lavage cytology in the treatment of locally advanced gastric cancer. Gastric Cancer 2007;10:29-34.

11. Aiko T, Sasako M. The new Japanese classification of gastric carcinoma: points to be revised. Gastric Cancer 1998;1:2530 .

12. Maruyama K, Kaminishi M, Hayashi K, Isobe Y, Honda I, Katai H, et al. Gastric cancer treated in 1991 in Japan: data analysis of nationwide registry. Gastric Cancer 2006;9:51-66.

13. Fujitani K, Yang H, Kurokawa Y, Park DJ, Tsujinaka T, Park BJ, et al. Randomized controlled trial comparing gastrectomy plus chemotherapy with chemotherapy alone in advanced gastric cancer with a single non-curable factor: Japan Clinical Oncology Group Study JCOG 0705 and Korea Gastric Cancer Association Study KGCA01. Jpn J Clin Oncol 2008;38:504-6.

14. Yamanaka T, Matsumoto S, Teramukai S, Ishiwata R, Nagai Y, Fukushima M. Analysis of risk factors for severe adverse effects of oral 5-fluorouracil S-1 in patients with advanced gastric cancer. Gastric Cancer 2007;10:129-34.

15. Kinoshita T, Nashimoto A, Yamamura Y, Okamura T, Sasako M, Sakamoto J, et al. Feasibility study of adjuvant chemotherapy with S-1 (TS-1; tegafur, gimeracil, oteracil potassium) for gastric cancer. Gastric Cancer 2004;7:104-9.

16. Kodera Y, Ito S, Mochizuki Y, Kondo K, Koshikawa K, Suzuki N, et al. A phase II study of radical surgery followed by postoperative chemotherapy with S-1 for gastric carcinoma with free cancer cells in the peritoneal cavity (CCOG0301 study). Eur J Surg Oncol 2009;35:1158-63.
17. Uedo N, Narahara H, Ishihara R, Takiuchi H, Goto M, Fjitani K, et al. Phase II study of a combination of irinotecan and S-1 in patients with advanced gastric cancer (OGSG0002). Oncology 2007;73:65-71.

18. Narahara H, Fujitani K, Takiuchi H, Sugimoto N, Inoue K, Uedo $\mathrm{N}$, et al. Phase II study of a combination of S-1 and paclitaxel in patients with unresectable or metastatic gastric cancer. Oncology 2008;74:37-41.

19. Yoshida K, Ninomiya M, Takakura N, Hirabayashi N, Takiyama W, Sato Y, et al. Phase II study of docetaxel and S-1 combination therapy for advanced or recurrent gastric cancer. Clin Cancer Res 2006;12:3402-7.

20. Cunningham D, Starling N, Rao S, Iveson T, Nicolson M, Coxon F, et al. Capecitabine and oxaliplatin for advanced esophagogastric cancer. N Engl J Med 2008;358:36-46.

21. Koizumi W, Takiuchi H, Yamada Y, Boku N, Fuse N, Muro K, et al. Phase II study of oxaliplatin plus S-1 as first-line treatment for advanced gastric cancer (G-SOX study). Ann Oncol 2010;21: 1001-5.

22. Chipponi J, Huguier M, Pezet D, Basso N, Hay JM, Quandalle P, et al. Randomized trial of adjuvant chemotherapy after curative resection for gastric cancer. Am J Surg 2004;187:440-5.

23. Bouché O, Ychou M, Burtin P, Bedenne L, Ducreux M, Lebreton $\mathrm{G}$, et al. Adjuvant chemotherapy with 5-fluorouracil and cisplatin compared with surgery alone for gastric cancer: 7-year results of the FFCD randomized phase III trial (8801). Ann Oncol 2005;16:1488-97.

24. Kochi M, Fujii M, Kanamori N, Kaiga T, Takahashi T, Kobayashi $\mathrm{M}$, et al. Neoadjuvant chemotherapy with S-1 and CDDP in advanced gastric cancer. J Cancer Res Clin Oncol 2006;132: $781-5$.

25. Satoh S, Hasegawa S, Ozaki N, Okabe H, Watanabe G, Nagayama $\mathrm{S}$, et al. Retrospective analysis of 45 consecutive patients with advanced gastric cancer treated with neoadjuvant chemotherapy using an S-1/CDDP combination. Gastric Cancer 2006;9:129-35. 